\title{
Estrategias de aprendizaje de los directores escolares en la sociedad del conocimiento
}

\section{School Principals' Learning Strategies in the Knowledge society}

Claudia Navarro-Corona

Profesora investigadora, tecnológico de Monterrey, Escuela de Humanidades y Educación, c.navarrocoronamail.com https://orcid.org/0000-0002-5077-8879

Resumen

La demanda de perfiles especializados, propia de la sociedad del conocimiento, ha permeado en los sistemas educativos latinoamericanos, por lo que se han establecido sistemas evaluativos que buscan garantizar cualidades específicas en los profesores y directores. La función directiva ha sido reconocida en la investigación educativa como una actividad relevante para el funcionamiento escolar y de forma indirecta para los resultados educativos; no obstante, en el caso de algunos sistemas educativos, por ejemplo, el mexicano, existen pocas ofertas formales para la preparación en esta función. En este marco, el objetivo del presente trabajo es identificar los mecanismos de formación a las que recurren los directores, así como los conocimientos que adquieren por medio de ellos. Por medio de una investigación cualitativa realizada mediante entrevistas a directores y autoridades del sistema educativo mexicano, se encontró que: 1) por medio de procesos de aprendizaje asistido y aprendizaje en la práctica, los directores se apoyan en figuras cercanas para adquirir conocimientos sobre la tarea; y 2) mediante procesos auto-administrados como la observación y reelaboración de modelos de dirección, estrategias de indagación, ensayo y error y consulta de materiales - los directores aprenden a identificar y solucionar problemas, relacionarse con otros, adaptarse a los contextos y administrar la documentación escolar. Se concluye que los saberes esenciales de la función directiva se adquieren en el campo de trabajo, por lo que se recomienda el rediseño de los esquemas formativos que estén centrados en la acción.

Palabras clave

Director escolar; aprendizaje del puesto; saberes de acción; socialización secundaria; sociedad del conocimiento.

\section{Abstract}

The demand for specialized profiles, typical of the knowledge society, has permeated Latin American educational systems, which is why evaluation systems have been established that seek to guarantee specific qualities in professors and scholar principals. The directive function has been recognized in educational research as a relevant activity for school functioning and indirectly for educational results; nevertheless, in the case of some educational systems, for example, the Mexican one, there are few formal offers for preparation in this function. In this framework, the objective of this paper is to identify the training mechanisms that directors use, as well as the knowledge they acquire through these ones. Through qualitative research conducted through interviews with directors and authorities of the Mexican educational system, it was found: 1) through assisted learning processes and learning in practice, in which the directors rely on nearby figures to acquire knowledge about their work; and 2) in self-adhered processes such as the observation and re-elaboration of management models, strategies of inquiry, trial and error and consultation of materials, principals apprehend to identify and solve problems, relate to others, adapt to contexts and manage school documentation. It is concluded that the essential knowledge of the directive function is acquired in the field of work, so it is recommended to redesign the training schemes that are focused on the action.

\section{Keywords}

School Principal; Job Training; Knowledge of Action; Secondary Socialization; Knowledge Society 


\section{Introducción}

Analizar la realidad desde el enfoque de la sociedad del conocimiento ha permito identificar una transformación en los modelos económicos, culturales y sociales. El concepto de sociedad de conocimiento refiere al entendimiento de que el conocimiento, junto con el capital y el trabajo, son los factores para el crecimiento y el desarrollo (Krüger, 2006). Este planteamiento apunta hacia una sociedad cientificada centrada en los servicios generados por el conocimiento especializado, más que por los productos y en la incorporación de innovaciones para obtener mejores resultados (Machlup, 1992).

Hablar de la definición de sociedad del conocimiento no refiere exclusivamente a la generación del conocimiento, sino a su distribución democrática, al fortalecimiento de sistemas autónomos y sostenibles de generación de conocimiento, y a la toma de decisiones basados en conocimiento científico. Es por esto que las decisiones políticas "dependen cada vez más de una legitimación científica" (Krüger, 2006, p. 5).

La cualidad del conocimiento se ha trasladado del ámbito del conocer, al saber hacer y saber ser, adquiriendo un enfoque de conocimiento competencial (Bozu y Canto, 2009). Si bien, la eficiencia de la escuela en una sociedad del conocimiento aún se encuentra en discusión (Ayustes, Gros y Valdivieso, 2012), al igual que el mercado y otros sectores, los sistemas educativos del mundo demandan, al menos en la política enunciada, perfiles especializados y legitimados por medio de procesos de evaluación. Tanto en Europa como en América Latina, los sistemas educativos han establecido sistemas de evaluación de profesores y directores con la finalidad de garantizar destrezas y conocimientos para la función (OCDE, 2013a, 2013b). En algunos países de América Latina y en especial en México, los resultados de las evaluaciones inciden en el ingreso a la función directiva y en la permanencia en el puesto y conservación del trabajo, adquiriendo rasgos de incertidumbre y dilemas que hasta entonces se habían mantenido al margen de las condiciones de trabajo del magisterio.

La relevancia que la figura del director tiene para la mejora escolar ha sido reconocida por la investigación educativa. Estudiosos del liderazgo y de la función directiva señalan que el trabajo del director afecta indirectamente el aprendizaje de los estudiantes (Leithwood, 1992, 2005; Branch, Hanushek y Rivkin, 2013). En el caso específico de México, hasta 2013, el ascenso de los profesores al puesto directivo se realizaba al participar y ganar en un sistema de promoción meritocrático en el que se obtenían puntos por cumplimiento de distintas tareas como la participación en actividades sindicales, participación en el mantenimiento del plantel escolar, formación general, disciplina y puntualidad (DOF, 1973). Con la aprobación de la Ley General del Servicio Profesional Docente (LGSPD); se modificaron los mecanismos de promoción. 
La LGSPD, además de regular los procesos de ingreso, permanencia y reconocimiento de la carrera de los profesores, estableció concursos de oposición para la promoción a los puestos directivos escolares y a otras posiciones de autoridad en el sistema educativo mexicano. Adicionalmente, en términos de esta Ley, se sustituyó el requerimiento de formación genérica por una formación específica en temas de liderazgo y gestión escolar (DOF, 2013).

Derivado de la aprobación de la LGSPD, se definieron perfiles parámetros e indicadores de la función directiva que definen la figura como un director que (1) conoce la escuela, su forma de organización y funcionamiento, (2) ejerce una gestión eficaz, (3) se reconoce como profesional de la educación y mejora continuamente, (4) asume y promueve los principios inherentes a su función y (5) conoce el contexto social y cultural de la escuela y colabora con la comunidad (SEP, 2017). También se enunciaron mecanismos de apoyo y soporte a la formación de los directores en formatos formales e informales; no obstante, en el país, estos continúan sin ser definidos ni operados regularmente.

La disposición permanente a buscar nuevas soluciones, propia de la sociedad del conocimiento, hace propicia una constante situación de riesgo en que las consecuencias de las innovaciones se extienden en la sociedad en su conjunto (Krüger, 2006). Con la finalidad de contrarrestar este efecto, se plantea que indagar sobre los conocimientos que se requieren para realizar la función directiva puede ayudar a construir una base más sólida en el campo de la formación de directores. Dado que los procesos de formación para los directores no se encuentran consolidados en México se plantean como preguntas de investigación ¿cómo aprenden los directores la función? ¿cómo se aprende a ser director? ¿quiénes enseñan?

El objetivo del presente trabajo es identificar los conocimientos que los directores refieren como elementales de la dirección escolar, así como los mecanismos de formación a los que recurren. Los resultados que aquí se reportan son parciales y forman parte de una investigación más amplia, ya concluida.

Además de la presente introducción, el trabajo se estructura en cinco partes. El segundo apartado, la fundamentación teórica, presenta dos conceptos centrales que contribuyen a la comprensión de las formas en las que los directores en México adquieren el aprendizaje de la función. Se incorporan los conceptos de saberes de acción, de aprendizaje en la práctica y de socialización secundaria. El tercer apartado, el método, expone el proceso por medio del cual se llegó a los hallazgos. El cuarto apartado presenta los resultados de la investigación. Muestra los elementos de conocimiento que son relevantes para la función directiva, desde la perspectiva de los participantes y explica los mecanismos por los cuales son aprehendidos. El apartado finaliza esclareciendo las relaciones que existen entre estos dos componentes. Finalmente, se presenta un apartado de conclusiones en las que se enuncian los hallazgos del estudio y se discuten a la luz de la producción científica en el tema. 


\section{Fundamentación teórica}

\subsection{Los saberes de acción en la función directiva}

Existe una amplia producción sobre las funciones de los directores escolares y los conocimientos sobre las funciones (Hales, 1986; Mintzberg, 1973; Leithwood, Harris y Hopkins, 2008; Sammons, Millman y Mortimore, 1998; Scheerens, 1992; Portner, 2005; Strong, 2009). En su postura más clásica, la investigación sobre las funciones de los directores son inventarios de tareas realizadas por estos actores. En este trabajo se retoma el concepto saberes de acción para revisar los conocimientos que los directores tienen en su función.

Los saberes de acción son los "conocimientos puestos al servicio de una lógica de acción en la que resulta dominantela preocupación deinnovar" (Gather,2003, p. 125). Este planteamiento es concordando con el enfoque de sociedad del conocimiento, en el que interactúan los conocimientos y las acciones para la mejora. Con frecuencia, las acciones realizadas por los directores se encuentran al servicio de distintos intereses. Un director escolar, por ejemplo, puede querer implementar una innovación, pero al mismo tiempo desear evitar conflictos con los profesores o con los padres de familia, querer parecer un líder moderno o aumentar su propia estima. En este medio, la movilización de conocimiento debe ocurrir sin excluir ningún interés en cuyo servicio se encuentra la función directiva.

Los saberes de acción emplean pragmáticamente el conocimiento, pues su lógica es multidimensional, al perseguir varios objetivos (Gather, 2003). Los orígenes de los saberes de acción también pueden ser diversos; pueden ser de carácter científico, estar arraigados en una cultura profesional o haber sido obtenidos por medio de una experiencia personal (Cros, 1993).

\subsection{Mecanismos de aprendizaje de la función}

En 2015 el informe Talis (Navarro-Corona, Martínez, Castro y Consuelo, 2015) mostró que el 76\% de los directores de secundaria participantes en el estudio reportó haber recibido formación específica sobre administración o dirección de escuelas, antes o después de ascender al puesto de director. Este porcentaje fue menor frente a la media de los países pertenecientes a la OCDE, en los que el 85\% de los directores reportó haber sido formado en administración o dirección de escuelas, antes o después de iniciar como director. No obstante, la investigación educativa documenta que la formación de los directores parece situarse más cercana a la formación informal que ofertas formalizadas para la preparación en las funciones del puesto de dirección. Se identifican dos formas en las que los directores aprenden la función. 
Una primera forma es el aprendizaje en la práctica. Desde esta postura, la producción científica sostiene que el desempeño en la función directiva requiere de experiencia ocupacional en el sistema escolar; es decir, el aprendizaje de la función directiva ocurre principalmente en la práctica, mientras el sujeto se desempeña en la función (Ortega, Castillo y Bettin, 2002), ya que ocupar el puesto directivo permite conocer las características de trabajo y el contexto. En esta experiencia se adquieren destrezas y habilidades que solo pueden ser desarrollados por este medio. Kolb (1984) y Carballo (2006) apoyan la postura del aprendizaje en la práctica y sostienen que el conocimiento se desarrolla cuando se realiza la actividad o se está en contacto directo con situaciones en las que la experiencia promueve el aprendizaje.

Si bien, los conceptos de aprendizaje y experiencia parecen estar relacionados en la adquisición de destrezas para la función directiva, Antunez y Gairín (2000) advierten que es un error asegurar que todas las experiencias desembocan en aprendizajes. La calidad de la experiencia, su continuidad y la oportunidad de reflexión son relevantes para lograr el aprendizaje. Una adición interesante es que, de acuerdo con Marcelo (1997), el aprendizaje en la práctica no ocurre aisladamente, sino que sucede en interacción con el contexto y con los individuos que participan en este.

Un segundo concepto que ayuda a comprender la forma en la que aprenden los directores es el de socialización. En términos de Berger y Luckmann (2003), la socialización es "la inducción amplia y coherente de un individuo en el mundo objetivo o de un sector de él" (p. 164) a fin de que internalice y aprehenda la significación de la sociedad en la que se encuentra. Hasta que el sujeto haya internalizado el mundo en el que se encuentra e interiorizado su cultura, entonces puede asumirse y ser asumido como miembro de una sociedad (Ortega y otros, 2002).

Berger y Luckmann (2003) distinguen dos tipos de socialización. La socialización primaria, que se desarrolla durante la primera incorporación del sujeto a la sociedad; es decir, durante la infancia. La socialización secundaria se presenta cuando el sujeto se desenvuelve en grupos a los que originalmente no pertenecía y aprehende los roles específicos, las manifestaciones rutinarias de comportamiento, el vocabulario empleado y las formas de interpretación propias de un grupo. Todos estos significados se construyen dialécticamente y son comunes todos sus miembros que pertenecen a la cultura de estos "submundos" institucionales (Berger y Luckmann, 2003).

A su vez, los estudiosos de la socialización dentro de las instituciones educativas desagregan el concepto en dos tipos: la socialización profesional, que refiere a los procederes adecuados en el marco de un puesto y a la adquisición de conocimiento y destrezas sobre la función que se desempeña (Staton, 2008 y Greenfield, 1985) y la organizacional que se vincula al conocimiento del funcionamiento de la institución y su contexto (Schvarstein, 1998 y Schein, 1971 y Greenfield, 1985). 
Tanto en la socialización como en el aprendizaje en la práctica, el sujeto se implica en experiencias "de primera mano" en la que los sujetos tienen la oportunidad de ocupar el puesto y realizar funciones propias de su posición (Wiendling y Dimmock, 2006). No obstante, existe una tercera noción de aprendizaje en la que el actor reelabora el conocimiento aprehendido cuando se encuentra expuesto a situaciones que puede observar, aunque no participe en ellas.

Marcelo (1995), Goodson (2004), Crow (2006) y Staton (2008) coinciden en que la socialización profesional de los directores no inicia con el ascenso, sino que suele iniciar años previos a la ocupación del puesto. En este mismo sentido, Wiendling y Dimmock (2006) refieren que la socialización profesional se manifiesta por medio de la observación de modelos -buenos o malos-, que permitan adquirir selectivamente valores, normas, actitudes y conductas de la profesión y construir una concepción de lo que significa ser director de escuela (Staton, 2008).

En cualquiera de los casos, la literatura documenta que los sujetos que se inician en una nueva función son altamente perceptivos (Schvarstein, 1998; 2003); no obstante, las formas en las que afronte el nuevo puesto repercutirá en otros procesos como la construcción del respeto, la autoridad y el clima de trabajo.

\section{Método}

La investigación se realizó desde el paradigma cualitativo, con enfoque interpretativo y con alcance comprensivo. El diseño de investigación se construyó tomando como referencia las aportaciones de McCurdy, Spradley y Shandy (2004) y Sandoval (2002), quienes definieron pasos para desarrollar el trabajo de campo y para conocer los fenómenos de estudio. La Figura 1 muestra una representación del proceso.

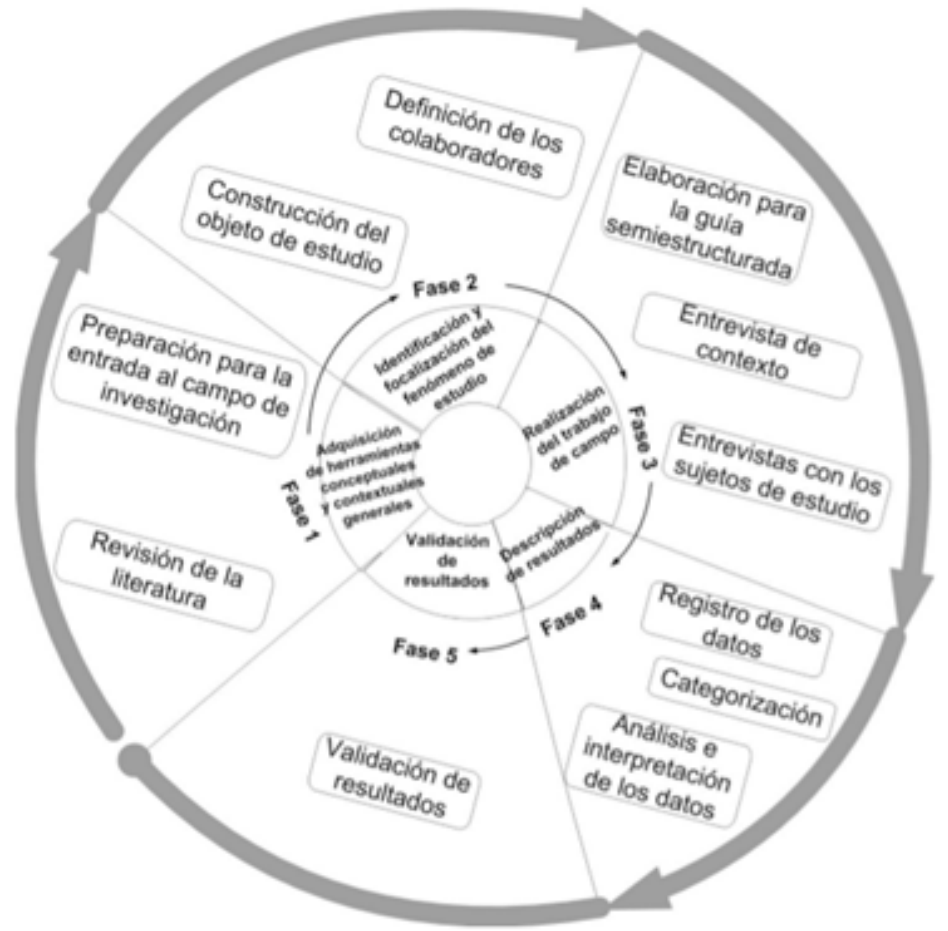


A continuación, se describe cada una de las fases seguidas en la investigación.

\subsection{Fase 1. Adquisición de herramientas conceptuales generales}

En esta fase se hizo la revisión de la literatura y la observación de entrevistas dirigidas por entrevistadoras experimentadas a actores provenientes del mismo contexto en el que se buscarían los participantes del estudio.

\subsection{Fase 2. Identificación y focalización del fenómeno de estudio}

La segunda fase consistió en la construcción del fenómeno de estudio. Esta se realizó a partir de entrevistas de contexto con autoridades educativas. Si bien esta fase no se reporta en el presente trabajo, se señala que el objeto de estudio de la investigación fue el trayecto laboral de los profesores para obtener el ascenso.

En esta etapa también se identificaron los participantes. De acuerdo con Merriam (1988), la selección de participantes se realizó por reputación. Se buscaba que fueran directivos expertos en el puesto, con conocimiento de la función directiva que pudieran explicar su proceso de formación y que desearan participar en el estudio.

Con este requerimiento, se solicitó orientación a las autoridades educativas, quienes sugirieron una lista de posibles participantes. Todos los directivos en la lista fueron contactados en sus escuelas e invitados a participar. Se garantizó la confidencialidad de datos personales y anonimato de sus respuestas.

Finalmente, el grupo de participación quedó integrado por cinco directores escolares. También participaron dos autoridades educativas del sistema educativo central estatal que participaron en entrevistas de exploración y de validación. Todos los participantes tenían más de 30 años de servicio y seis de los siete participantes contaba con estudios en magisterio, egresado de alguna institución formadora de maestros en México.

\subsection{Fase 3. Realización del trabajo de campo}

La recogida de información se realizó por medio de la técnica de entrevista semiestructurada. El guion fue construido de manera iterativa, ya que, después de cada entrevista, era ajustado para incorporar preguntas que indagaran sobre información novedosa que se identificaba en cada entrevista. Este procedimiento de construcción permitió corroborar en cada entrevista la información identificada y llegar a la saturación de las categorías en la fase de análisis, de acuerdo con lo recomendado con Strauss y Corbin (2002). 


\subsection{Fase 4. Descripción de resultados}

Para el registro de la información se siguieron las recomendaciones de Bertely (2000) y Denzin (2009). Las entrevistas fueron audiograbadas y transcritas en su totalidad. Cada entrevista tuvo una duración aproximada de 80 minutos. Las transcripciones produjeron un corpus de 180 páginas en interlineado 1.5 y letra número 12. Se asignó un código de identificación a cada entrevista integrado por el tipo de entrevista pseudónimo de participante, página y fecha del levantamiento.

El análisis de resultados se realizó conforme a los parámetros de Woods (2010) y Strauss y Corbin (2002). En un primer momento se realizó un análisis especulativo en el que tras una primera lectura se registraron dudas, se identificaron datos incompletos o contradictorios y se identificaron primeras similitudes entre las declaraciones y testimonios de los participantes.

En un segundo momento, se eligieron las unidades de análisis seleccionándolas por unidades de significación. Las unidades se etiquetaron con códigos. Se buscó que estos fueran excluyentes. Se obtuvieron 180 códigos específicos.

Posteriormente, los códigos fueron organizados en categorías. Se requirieron varias revisiones para obtener un sistema de códigos y categorías que cumplieran los criterios de exclusión definidos. En el tercer momento se realizó un análisis axial en el que se identificaron las relaciones entre las categorías. Se obtuvieron 27 categorías organizadas en ocho familias.

\subsection{Fase 5. Validación de resultados}

Para validar los resultados de la investigación se empleó la técnica de feedback descrita por Miles y Huberman (1996), la cual consiste en regresar información a los actores del contexto estudiado para que corrijan, precisen, descarten o aprueben las conclusiones a las que el investigador ha llegado.

Los participantes validadores participaron también en entrevistas de contexto para la construcción del objeto de estudio. Se buscó que los participantes tuvieran amplio conocimiento del sistema educativo y del puesto. Se realizaron dos entrevistas de validación, en ambas se aprobaron los resultados. No se reportaron resultados específicos de esta fase, sino que se precisaron las interpretaciones realizadas en el análisis.

\section{Resultados}

Los resultados aquí reportados corresponden a la categoría "Aprendizaje de la función directiva"; una de las siete categorías que conformaron la familia "Aprender a ser director". A partir del análisis 
inductivo, dicha categoría se definió como los elementos del proceso no formal de aprendizaje en el que el directivo se involucra para apropiarse de conocimientos y habilidades para el desarrollo de sus funciones. En esta categoría, además de identificar los saberes de la función directiva, los participantes compartieron los distintos mecanismos que ponen en práctica para el aprendizaje. Desde la perspectiva de los participantes existen cuatro elementos de aprendizaje relevantes. Estos conocimientos solo son adquiridos cuando el sujeto se encuentra en la experiencia o expuesto a esta. Estos son:

1) Identificación y solución de problemas específicos. Para los participantes, implica la habilidad de detectar situaciones problemáticas o potencialmente problemáticas para solucionarlas o prevenirlas.

2) Relación con los integrantes de la comunidad educativa. Es el desarrollo de la habilidad para relacionarse de manera adecuada con los distintos actores que se relacionan en el contexto del plantel escolar: alumnos, docentes, padres de familia u otros.

3) Adaptación a distintas situaciones y contextos. Consiste en desarrollar la habilidad de cambiar las estrategias de solución según los requerimientos de situaciones.

4) Administración y control de la documentación escolar y del centro educativo. Refiere al aprendizaje en el manejo de todo tipo de documentación de la escuela y el registro de información, así también de la organización y administración de la escuela y sus recursos.

Estos cuatro campos de conocimiento están regulados por la normatividad y el contexto. Así, el directivo debe aprender dichas normas de acción e involucrarse en un proceso de aprendizaje en la práctica, no solo para desarrollar las habilidades requeridas para el puesto, sino para conocer cómo es que deben hacerse las cosas (Berger y Luckmann, 1998).

El acceso a estos conocimientos ocurre al poner en práctica mecanismos para acceder al conocimiento. Estas formas no son excluyentes, sino que el actor las intercala, las sustituye, las complementa o las mezcla. Teóricamente, se pueden distinguir dos formas de acceder al conocimiento de la función directiva. La primera es el aprendizaje asistido, que requiere de la asesoría de otros miembros de la comunidad educativa. La segunda es el aprendizaje autodirigido, mismo que se desarrolla mediante la implementación de estrategias de aprendizaje autónomo

\subsection{Aprendizaje asistido}

Aun cuando se han institucionalizado procesos de evaluación, la preparación formal para el trabajo directivo es limitada. Dada esta condición, los directores escolares inician procesos de interacción social donde adquieren los conocimientos para realizar sus funciones. El total de directivos entrevistado señaló que se va "aprendiendo con la práctica diaria" (E-I-Armando-161009-12). Es "como la canción", 
señala un directivo entrevistado, "se hace camino al andar" (E-I-Juan-151009-15). Así se va aprendiendo a ser directivo.

Los entrevistados comentan que "la práctica hace al maestro, la escuela te da la teoría, te da todo, pero donde te formas es en la práctica" (2E-I-Carlos-301009-9) y que "nosotros nos hicimos, nos certificamos en base al trabajo que realizábamos" (E-C-Rosa-220509-19). Así, además de representar una importante fuente de aprendizaje, la práctica en el cargo legitima los saberes de los directivos ante la comunidad escolar e, incluso, ante sí mismos.

Los participantes mencionan distintos actores con los cuales, en su rol de directivo, han establecido relación para aprender la función. Estos actores fungen como figuras de apoyo de los recién ascendidos que guían para la para la adquisición de conocimientos específicos. La figura de apoyo que los entrevistados refieren con mayor frecuencia son "las secretarias que conocen [las funciones] a través de su práctica y los van orientando" (2EI-Carlos-301009-9, 10). "Nos hemos dado cuenta que

(...) como ellas sí están desde que entran, si tienen un año, dos años, tres años, cinco años saben el manejo" de la escuela (E-I-Juan-151009-16). Aunque las secretarias son referidas como el principal apoyo al aprendizaje de los directivos, los entrevistados aclaran que ellas apoyan con lo referente a la administración de la documentación escolar y del centro educativo.

Existen otras figuras de apoyo que son mencionadas por los entrevistados mediante el proceso de socialización. Los inspectores y otras autoridades del sistema educativo son mencionados como actores que asesoran o dan instrucción, indicaciones y respuestas en apoyo a ciertas situaciones específicas por las que atraviesan los directores, aunque en menor medida; por otro lado, los orientadores y prefectos otorgan apoyo y los asesores técnico-pedagógicos dan sugerencias de menor impacto.

\subsection{Aprendizaje autodirigido}

Como parte del aprendizaje de la función, los participantes refirieron la observación de modelos directivos en los años en los que trabajaron como docentes. El desempeño de buenos y malos directores sirve de modelaje de la función. Como proceso de aprendizaje, este mecanismo requiere del observador la habilidad para discriminar las experiencias a las que estuvo expuesto y en las que observó el desempeño de la función directiva. Se trata de una tarea selectiva en la que los sujetos identifican prácticas de la función y formas de ser director. Uno de los directores entrevistados señaló:

"Lo que yo detestaba de mis directivos si quiero problemas pues voy a seguir haciendo lo que a mí me hacían. Si no quiero problemas y quiero que mi centro educativo funcione voy a desechar los grandes errores que yo marqué a un directivo. Si yo trate mal a un directivo [por la manera en que actuaba] pues 
yo creo que me van a tratar mal a mí haciendo eso [que él mismo hacía] (...) La experiencia me dice que yo no quiero ser de esta forma, quiero ser de esta otra y ponemos en práctica todo lo que en nuestro andar recogimos, de lo bueno y lo malo" (E-I-Juan-151009-116).

El contacto con el puesto puede ser de forma más cercana, esta condición se vincula a la estrechez en los vínculos entre el director y el aspirante. Un testimonio de los entrevistados da cuenta de esto: uno aprende, todos los días va aprendiendo de tener una directora que también deja enseñanzas: «mire profe, las cosas así» y así uno va copiando modelos y ajustándolo a la [propia] visión" (1E-ICarlos-150609-15).

Un segundo mecanismo de aprendizaje es la autorregulación de la información. Los directores ponen en práctica estrategias para el aprendizaje de la función y la internalización de las características de la organización. Estas estrategias son autorreguladas por los directores y están encaminadas a aproximarse a los significados manejados por el medio en donde trabaja. A continuación, se especifican las prácticas de aprendizaje referidas por los participantes.

Ensayo y error. La primera estrategia referida por los participantes es lo que denominan la "prueba de ensayo y error". Desde la perspectiva de los directivos entrevistados, la prueba de ensayo y error es útil para detectar opciones de solución adaptadas a distintos contextos de trabajo. Dicen: "lo que me sirvió en aquella escuela aquí ya no me funciona, tengo que ir modificando, manejando nuevas alternativas (...). Tenemos que cambiar algunas cosas; [además] los errores son aprendizajes. Son enseñanzas. Eso es. Así nos vamos haciendo los directivos" (1E-I-Carlos-150609-16, 17).

Elaboración de preguntas. Esta es una estrategia relacionada con la interacción entre el directivo y alguna figura de apoyo para el aprendizaje. Los participantes dicen que "yo puedo preguntarle al inspector, puedo preguntarle al sistema educativo y ellos me sacarán de duda" (E-I-Armando-161009-117).

Autoanálisis. Se considera una estrategia de exploración de la propia práctica profesional para detectar errores y fallas. Los entrevistados dicen: "todos los días hay que ir modificando las expectativas, viendo, analizando qué hice bien, qué hice mal, qué puedo cambiar" (1E-I-Carlos-150609-16).

Autodidactismo. Refiere al uso de materiales para aprender las funciones directivas. Esta estrategia es mínimamente referida por los entrevistados. Ellos señalan que el único material de aprendizaje son los instructivos; a estos se acude principalmente cuando se tienen dudas sobre la administración de documentación específica.

Estos procesos de aprendizaje asistido y autorregulado no son excluyentes. La Figura 2 muestra una representación de los elementos de aprendizaje y los mecanismos que los directores ponen en juego para aprehenderlos. 


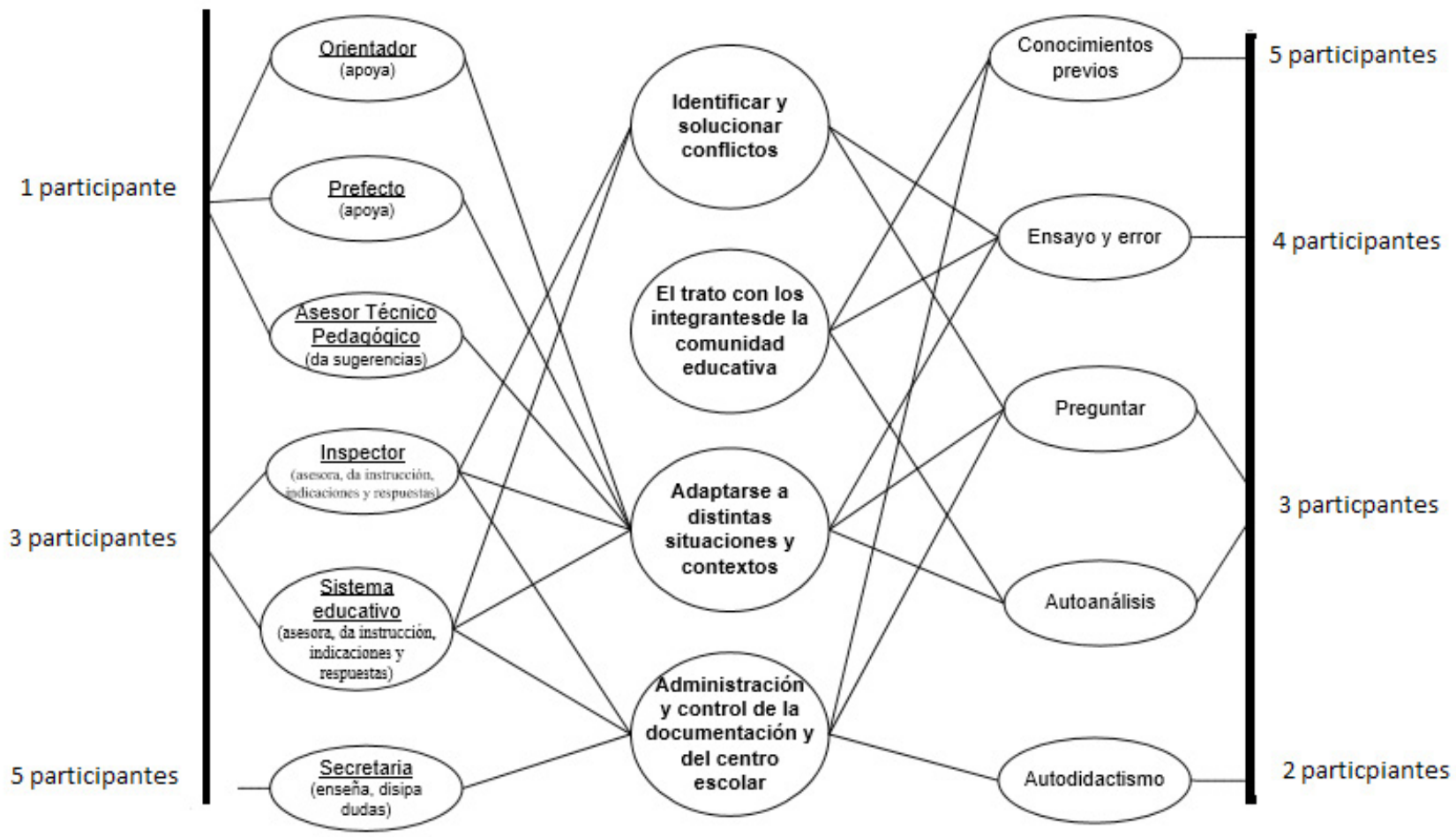

Figura 2. Relaciones entre los elementos de conocimiento y los mecanismos de aprendizaje de los directores escolares Descripción: El análisis de los testimonios de los participantes permitió a identificación de relaciones entre los elementos de conocimiento y los mecanismos de aprendizaje. Cada línea representa una mención de un participante. Fuente: Elaboración propia.

La revisión de los testimonios aportados por los participantes permitió identificar relaciones entre los elementos de conocimiento y los distintos mecanismos que los directores mexicanos siguen para aprender las funciones. Adicionalmente, se identificó el número de participantes que mencionó cada forma de aprendizaje, lo que ilustra la naturaleza de la función directiva y la forma en que los directores identifican la relación entre los conocimientos y las figuras de apoyo al aprendizaje.

\section{Conclusiones}

Existe un conjunto de saberes esenciales de la función directiva que son transversales a las distintas funciones de un director escolar. Los participantes reportan la identificación y solución de problemas, las formas de relacionarse con los miembros de la comunidad educativa y la adaptación a distintas situaciones y contextos como saberes que se enfocan en la operación para la realización de la función directiva. Estos elementos de conocimiento difícilmente pueden ser adquiridos en proceso formales de formación.

El planteamiento de que adquisición de conocimiento en la práctica no es nuevo. Autores como Kolb (1984), Marcelo (1997) Ortega, et al. (2002), Carballo (2006) han planteado que el aprendizaje de la 
función solo es posible en la práctica. Los resultados de este estudio confirman las aportaciones de estos autores. Así también, la advertencia de Antunes y Gairín (2000) de que la experiencia debe ser de calidad para tener efecto en el aprendizaje cobra relevancia. En este sentido, es posible proyectar que la innovación de esquemas de formación que se realicen en la práctica en la que se considere la participación de actores cercanos a la labor de los directores podría propiciar experiencias profesionales y personales vinculadas a la función que faciliten el aprendizaje de la función directiva.

Los saberes esenciales de la función directiva se originan en la experiencia o en el conocimiento de un grupo profesional y no propiamente en procesos científicos de generación de conocimiento. Los participantes refieren la práctica profesional y la experiencia personal como la fuente de sus saberes. Cross (1993) y Gather (2003) también encontraron que saberes de acción, como los de la dirección escolar, pueden tener distintos génesis, pero están orientados hacia la innovación en la función. Bozu y Canto (2009) refirieron que, en la sociedad del conocimiento, el mero acto de conocer no es suficiente, sino que es necesario haber y saber ser para movilizar el conocimiento. En este sentido, las prácticas de los directores, si bien no parecen tener el mismo nivel de inmersión en la sociedad del conocimiento que otros campos como el mercado y la empresa, sus prácticas parecen estar permeadas por las características de este paradigma, por lo que los saberes se orientan hacia la movilización para la acción.

La observación y reelaboración de modelos realizados por los aspirantes al puesto permite construir una idea de lo que significa ser director y la orientación de la función directiva. Lo participantes reportan como referencias de su propia práctica el aprendizaje de modelos positivos o la reelaboración de modelos negativos. Wiedling y Dimmock (2006) refieren la observación como el mecanismo para adquirir valores sobre la profesión, actitudes y conductas. Este conocimiento sobre el aprendizaje de los directores podría orientar el diseño de experiencias formativas innovadoras.

Se encontró que la naturaleza de la función directiva en México sigue siendo esencialmente burocrática y de control. Los principales requerimientos de información que los directores hacen a las figuras de apoyo se centran en los asuntos de control administrativo, por lo que las secretarias se identifican como principales figuras de apoyo al aprendizaje. Esto ilustra las demandas de la tarea directiva. Berger y Luckmann (2003) señalaron que la socialización secundaria ocurre por medio de la interacción con otros. Los hallazgos del muestran que la función directiva continúa en un marco de control administrativo, que necesita ser repensado.

Por último, se identifica la necesidad de pensar en formatos más flexibles de formación que se desarrollen en los espacios profesionales y que desarrollen cualidades específicas en los perfiles profesionales de los directores. 


\section{Referencias}

Antúnez, S. y Gairín, J. (2000). La acción directiva. En La organización escolar. Práctica y fundamentos (pp. 235-247). Barcelona: Graó

Ayuste, A., Gros, B. y Valdivieso, S. (2012). Sociedad del conocimiento. Perspectiva pedagógica. Ponencia presentada al XXXI Seminario Interuniversitario de Teoría de la Educación, "Sociedad del Conocimiento y Educación». Plasencia, 11-14 de noviembre (pp. 1-31).

Berger, P. y Luckmann, T. (2003). La construcción social de la realidad. Buenos Aires: Amrrurtu editores.

Bertely, M. (2000). Conociendo nuestras escuelas. Un acercamiento etnográfico a la cultura escolar. México: Paidós.

Bozu, Z. y Canto, P. J. (2009). El profesorado universitario en la sociedad del conocimiento: competencias profesionales docentes. Revista de formación e innovación educativa universitaria, 2(2), 87-97. Recuperado de: goo.gl/h8TZCU

Branch, G., Hanushek, E. y Rivkin, S. (2013). School Leaders Matter: Measuring the Impact of Effective Principals. Education Next, 13(1), 63-69.

Carballo (2006). Aprender haciendo. Guía para profesores. Aproximación a los espacios de aprendizaje basados en la acción, la experiencia y el grupo de trabajo y aplicaciones prácticas. Recurso electrónica del II Encuentro sobre experiencias grupales e innovadoras en la docencia universitaria. Madrid: Centro de Estudios Superiores Felipe II, campus Aranjuez, Universidad Complutense de Madrid.

Cross, F (1993). L'innovation a l'école: forces et illusions. París. PUF.

Crow, G. (2006). Complexy and the beginning principal in the United States: perspectives on socialization. Journal of Educational Administration, 44(4), 310-325. doi:https://doi.org/10.1108/09578230610674930 Denzin, N. (2009). The research act in sociology: A Theorical Introduction to Sociological Methods. Londres: Butterworth

DOF. (2013). Decreto por el que se expide la Ley General del Servicio Profesional Docente. Recuperado de: goo.gl/JG1E9a

Garther, M. (2003). Saberes de acción, saberes de innovación de los directores de centros. En G. Pelletier (Coord.). Formar a los dirigentes de la educación. Madrid: La Muralla.

Goodson, I. F. (2004). Historias de vida del profesorado. Barcelona: Octaedro. 
Greenfield, W. D. (1985). Studies of the assistant principalship: toward new avenues of inquiry. Education and Urban society, 18(1), 7-27. doi:https://doi.org/10.1177/0013124585018001002

Hales, C. P. (1986). What do managers do? A critical review of the evidence. Journal of Management Studies, 23, 88-115. doi:https://doi.org/10.1111/j.1467-6486.1986.tb00936.x

Kolb, D. (1984). Experiential learning. Experience as the source of learning and development. New Jersey: Prentice Hall.

Krüger, K. (2006). El concepto de sociedad del conocimiento. Revista bibliográfica de geografía y ciencias sociales, 683(11), 1-14. Recuperado de: goo.gl/qS1hdt

Leithwood, K. (1992). The Move Toward Transformational Leadership. Transforming Leadership, 49(5), 8-12.

Leithwood, K., Harris, A. y Hopkins, D. (2008). Seven strong claims about successful school leadership. School leadership and management, 28(1), 27-42. doi:https://doi.org/10.1080/13632430701800060

Machlup, F. (1962). The production and distribution of knowledge in the United States (Vol. 278). Princeton university press. Recuperado de: goo.gl/cuAjkL

Marcelo, C. (1995). Formación del profesorado para el cambio educativo. Barcelona: EUB.

Marcelo, C. (1997). Evaluación y modelos para la formación continua de los formadores. Universidad de Sevilla. Recuperado de: http://prometeo.us.es/recursos/seminario/marcelo.doc.

McCurdy, D. W., Spradley, J. P. y Shandy, D. J. (2004). The Cultural Experience: Ethnography in Complex Society. Tennesse: Sage.

Merriam, S. B. (1988). Defining a Research Problem and Selecting Case. In Case Study Research in Education. A Qualitative Approach (pp. 36-52). San Francisco: Jossey-Bass.

Miles, M. B. y Huberman, A. M. (2010). Qualitative data analysis: An expanded sourcebook (2a ed.). Thousand Oaks: Sage Publications.

Mintzberg, H. (1973). The nature of managerial work. New York: Harper \& Row.

Murillo, J. (2007). Evaluación del desempeño y carrera profesional docente. América y Europa. Santiago: UNESCO.

Navarro-Corona, C., Martínez, S. Castro, D y Consuelo, S. (2015). Gestión escolar y liderazgo de los directores en E. Backhoff y J. C. Pérez-Morán (Coords.), Segundo Estudio Internacional sobre la Enseñanza y el Aprendizaje (TALIS 2013). Resultados de México. México: INEE. Recuperado de: https:// www.oecd.org/edu/school/Mexico-TALIS-2013_es.pdf 
OCDE. (2013a). Synergies for Better Learning: An International Perspective on Evaluation and Assessment. OCDE Pubishing.

OCDE. (2013b). Teachers for the 21st Century: Using Evaluation to Improve Teaching. OECD Publishing. Ortega, F., Castillo, J. y Bettín, G. (2002). Fundamentos de sociología. Madrid: Editorial Síntesis.

Portner, H. (2005). Teacher Mentoring and Induction. The state of the art and beyond. California: Corwin Press.

Sammons, P., Hillman, J. y Mortimore, P. (1998). Características clave de las escuelas efectivas. México: Secretaría de Educación Pública.

Sandoval, C. A. (2002). Investigación cualitativa. Bogotá: Instituto Colombiano para el Fomento de la Educación Superior.

Scheerens, J. (1992). Effective schooling. Londres: Cassell.

Schein, E. H. (1971). The individual, the oganization, and the carrer: a conceptual scheme. The journal of Applied Behavioral Science, 7(4), 401-426. doi:https://doi.org/10.1177/002188637100700401

Schvarstein, L. (1998). Diseño de organizaciones. Tensiones y paradojas. Buenos Aires: Paidós.

Schvarstein, L. (2003). Inteligencia Social de las Organizaciones. Buenos Aires: Paidós.

SEP [Secretaría de Educación Pública]. (2017). Perfiles parámetros e indicadores para el personal con funciones de dirección, de supervisión y de asesoría técnica pedagógica en educación básica. Recuperado de: goo.gl/CT3Bps

Staton, A. Q. (2008). Teacher Socialization. The international Encyclopedia of Communication. Wolfgang: Donsbach. doi:https://doi.org/10.1002/9781405186407.wbiect016

Strauss, A. y Corbin, J. (2002). Bases de investigación cualitativa. Técnicas y procedimientos para desarrollar la teoría fundamentada. Medellín: Universidad de Antioquía-Contus.

Strong, M. (2009). Effective teacher induction and mentoring: assessing the evidence. New York: Teacher College Press, Culumbia University.

Weindling, D. y Dimmock, C. (2006). Sitting in the "hot seat": new headteachers in the UK. Journal of Educational Administration, 44(4), 325-340. doi:https://doi.org/10.1108/09578230610674949

Woods, P. (2010). La escuela por dentro. La etnografía en la investigación educativa. Barcelona: Paidós. 